\title{
A Network of FLOSS Competence Centres
}

\author{
Jean-Pierre Laisné ${ }^{1}$, Nelson Lago ${ }^{2}$, Fabio Kon $^{3}$, and Pedro Coca ${ }^{4}$
}

1 Open Source Strategy Director for Bull, President of OW2 Consortium Coordinator of Qualipso Competence Centres, Coordinator of 2020 Floss Roadmap

$$
\begin{gathered}
\text { http://www.ow2.org/ } \\
\text { http://www.qualipso.org } \\
\text { http://2020Flossroadmap.org }
\end{gathered}
$$

2 Techical director at the Centro de Competência em Software Livre Qualipso at University of São Paulo http://ccsl.ime.usp.br

3 Professor at the Department of Computer Science at, University of São Paulo Head of Centro de Competência em Software Livre Qualipso at University of São Paulo http://www.ime.usp.br http://ccsl.ime.usp.br

4 Univeristy Rey Juan Carlos at Madrid, Morfeo Qualipso Competence Centre http://www.urjc.es/ http://cc.libresoft.es/

\begin{abstract}
The goal of a Network of Competence Centers is to provide to FLOSS users, developers, and consumers, high-quality resources and expertise on the various topics related to FLOSS. This may be achieved via education, training, consulting, hosting, and certification not only in terms of tools and platforms but also methodologies, studies, and best practices. Based on the experience of QualiPSo Competence Centres, we observe how such a Network is working as a mechanism for sharing success stories, failures, questions, recommendations, best practices, and any kind of information that could help the establishment of a solid international collaborative environment for supporting quality in FLOSS. New Competence Centres are invited to the QualiPSo Network after their proposals are evaluated by the QualiPSo Competence Centres Board to ensure that the prospective Competence Centre is compliant with the QualiPSo Network Agreement, sharing a common vision and ethics. Each Competence Centre acts in its geographical region to increase the awareness of FLOSS and to better prepare the IT workforce for developing and using FLOSS based solutions. As of 2009, the process for Competence Centre creation is sustainable and reusable; guidelines for establishing proposals and opening new Competence Centres have been created, and promotion of Qualipso Competence Centres is done world wide from India to USA thanks to key initiatives such as the Open World Forum and the FLOSS Competence Centre Summit. This lecture will expose how these Competence Centres relate to each other, which governance model is used and, based on existing experiences, will describe how they currently operate in Europe and Brazil and what is planned in Italy, Belgium, Japan, and China for 2010.
\end{abstract}




\section{FLOSS Adoption: It's All about TRUST}

There are significant signs of broad dissemination of FLOSS concepts both within industries and within governments. Nevertheless, there is still reluctance to massive adoption of FLOSS development, mainly due to lack of confidence. Several "grey areas" around FLOSS cause concerns: legal uncertainties, such as IP protection and indemnification; quality guarantees, such as development life cycle, documentation, support, reliability, and performance; and, finally, business issues such as business models capable of maintaining sustainability.

All these concerns can be summarised in one word: trust. And trust is not a quality that can be claimed without being proved. It also relies on perception, on non technical questions such as "Who is behind FLOSS?", "Why be confident in FLOSS?", or even "How to be confident in FLOSS?".

\subsection{A Distributed Network of Trustworthy and Highly Skilled Resources}

It is commonly known that "people talk to people". We want to make use of this fact to establish confidence in FLOSS by offering independent and qualified support and services by means of Competence Centres disseminated around Europe, Brazil, and China. Interconnected among them, these Competence Centres represent a network that openly offers access to skilled resources and promotes trust in FLOSS: It enables end users, ISVs (Independent Software Vendors), developers, etc. to find answers to their questions and use FLOSS in their operations in a reliable and trusted manner.

These services are delivered thanks to collaborative platforms, tools, and process developed mainly by the QualiPSo project1. Each Qualipso Competence Centre, having a basic set of functionalities, represents an aggregation point of technologies, skills, and policies. While these competence centres may differ one from the other by their level of expertise on specific domains, all together the Qualipso competence centres form a network of expertise sharing the same ethics and values.

\section{$1.2 \quad$ In FLOSS We Trust}

Each competence centre is a set of physical resources (bricks \& mortar) and virtual sites dedicated to maximize the reusability of tools, processes, and shared knowledge by offering services and training. These services will make documents, tools and platforms openly available to organizations needing to assess the legality, sustainability, robustness, and interoperability of their critical applications running on top of FLOSS.

Qualipso Competence Centres will also favor the reusability of the results of the R\&D effort of the Qualipso Project not only in terms of tools and platforms

\footnotetext{
${ }^{1}$ A research project that aims to work on several issues related to FLOSS in order to foster its adoption by the industry at large.
} 
but also of studies, best practices, and other miscellaneous information. They will make these results available to both Community and Industry and will promote them at large through communication programs (public and press relations, white papers, seminars, courses, workshops, conferences, etc.).

The Qualipso Competence Centres are designed to be replicable but replication can only work if all components of the competence centre are reusable from legal to technical aspects. Therefore, all information concerning competence centres is documented and this documentation is freely available to anyone. Transparency of the process must insure that there is neither hidden agendas nor Trojan horses in the competence centre model. The replication aims to provide in each country the same level of information, the same homogeneous tools and processes, and also some dedicated local resources which act as national FLOSS experts specialized on identified specific topics.

QualiPSo competence centres are to be distributed worldwide, and may be instantiated by geographical location, e.g., Paris, Berlin, Madrid, São Paulo, Tokyo, Guangzhou; or by organizations, such as OW2, Morfeo; or even by large companies, such as Telefonica or eGovernment-oriented companies such as SERPRO in Brazil.

\section{General Description}

The goal of the Qualipso Network is to federate all Qualipso Competence Centres sharing the same vision, goal, ethics, methods, and tools. So, at the heart of the Network, stands the Qualipso Network Agreement. This document describes how these principles are implemented and how all components of the Qualipso Network are governed.

Each Competence Centre has to define its policy concerning aspects such as its mission statement and scope, market and geographical area of interest, technological specialization, legal and funding model, communication plan etc. A more detailed description will be given during the lecture for attendees to evaluate the compliance of these elements with the FLOSS culture. This description is based on the content of Qualipso Network Agreement avilable at http://www .qualipso.org/sites/default/files/Qualipso — D8.2 Network Agreement V1.17.pdf.

\subsection{Structure of the Network and Its Components}

At the most basic level, the Qualipso Network represents the network of Qualipso Competence Centres. It is a distributed network of trustworthy and highly skilled resources that reuse technologies, procedures, and policies produced by the Qualipso Project. The Qualipso Network federates all Competence Centres and its goal is to protect Qualipso commonly produced assets, intellectual property, and brands. At its head, we find the Qualipso Network Board, which is composed of representatives of all Qualipso Competence Centres. This board manages conflicts of interest and decides on matters related to: registration/cancellation of 


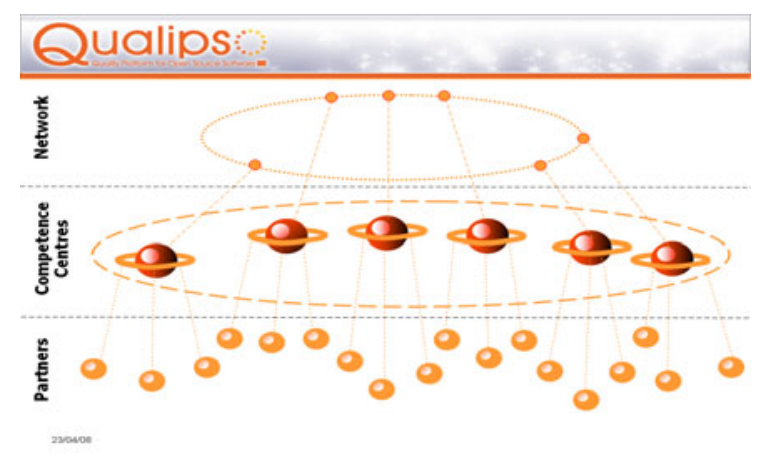

Fig. 1. Qualipso competence centres network architecture

Competence Centres, protection of common assets, approval of new Qualipso services, and updates of the Qualipso Network Agreement.

At the next level, Qualipso Competence Centres facilitate reusability of the results of the Qualipso project. Competence Centres provide expertise and services in the form of a set of independent and vendor neutral basic services, tools, and methods on topics addressed by the Qualipso Project from legal advice to benchmark results. Specific services may also be created. Each Competence Centre shall have its own legal model in compliance with defined principles, in order to ensure sustainability of the activities, fairness among partners, openness to new partners, and to define liabilities, responsibilities, and territory. Each Competence Centre shall also comply with the Qualipso Network Agreement and be self-sustainable, i.e., manage its own revenues, define its own funding model, and ensure its efficiency. In short, Competence Centres act locally and cooperate globally.

Finally, Partners are organizations that decide to create a Competence Centre according to some common interest (geography, language, technology, etc.). Partners are committed to provide the necessary resources for each Competence Centre to achieve its goals. There are two types of Partners: Active Partners, which contribute to daily activities, and Associate Partners, which act as sponsors.

\subsection{Benefits of Belonging to the Qualipso Network}

As part of the Qualipso Network, Competence Centres are able to share success stories, failures, questions, recommendations, best practices, and any kind of information that could help the establishment of a solid international collaborative environment for supporting quality Open Source Software. Therefore, Competence Centres and their worldwide network will support the continuing development of the Qualipso vision, helping to provide a sustainable quality platform for Open Source Software.

To summarize, the key benefits of the Qualipso Network of Competence Centres are: 


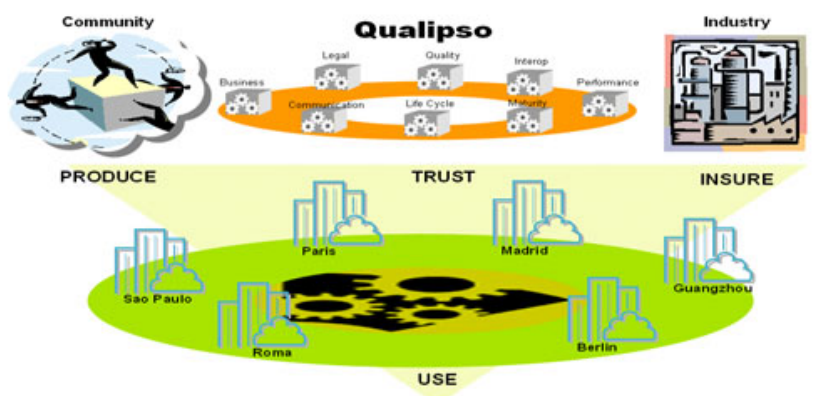

Fig. 2. Interaction between FLOSS comunities, the QualiPSo research project, industry, and competence centres

- Global synergies on marketing and communication.

- Multinational shared expertise and knowledge base.

- Local presence through a global network and access to different markets.

\section{Competence Centres at Work}

The Qualipso Competence Centre in Spain (http://cc.libresoft.es/) is a joint effort of the GSyC/LibreSoft research group at the Universidad Rey Juan Carlos (URJC), the CETTICO research group at the Universidad Politécnica de Madrid (UPM) and Telefonica R\&D. These are founding members of the MORFEO community, with which the Competence Centre shares a close link. Teófilo Romera Otero, from the GSyC/LibreSoft group, states: "The creation of the Competence Centre is an opportunity to uplift our activities to a higher and institutionalized level, allowing a better impact for the creation of knowledge and the services we have been already offering for years".

The Qualipso Competence Centre in Brazil (http://ccsl.ime.usp.br) is located at the University of São Paulo and started its activities in December, 2008. According to Prof. Fabio Kon, the São Paulo Competence Centre director, "the Competence Centre is an excellent means for the university to communicate with the Brazilian society and software industry; it contributes not only sharing the knowledge developed by our research groups but also working as a meeting point for students, researchers, and practitioners from our region and abroad; we also expect to benefit from being part of an international collaborative network focusing on the quality of Free and Open Source Software".

The German Competence Centre is located at the Fraunhofer Institute for Open Communication Systems (FOKUS) in Berlin and started its activities in the first quarter of 2009. Its main emphasis is on providing a factory for FLOSS projects and on Qualipso services in the context of technical, semantic, and organizational interoperability between Open Source systems as well as between Free/Libre/Open Source systems and closed source systems. Governmental organizations as well as industry and research will benefit from these contributions and the Qualipso network. 


\section{Beyond Qualipso Competence Centres: FLOSS CC Manifesto}

As a result of the First International FLOSS Competence Centres Summit, organized by Qualipso as part of the Open World Forum, 11 leading FLOSS promotion organizations worldwide (Berlin, Chennai, Guangzhou, Madrid, Maribor, Newry, Paris, Portadown, Raleigh, São Paulo, Tokyo) joined forces to create a solid international network of FLOSS Competence centers to share experiences, define best practices, strengthen synergies, and collaborate on the promotion of FLOSS.

As a first step, this network provides a Manifesto for FLOSS Competence Centres, written and signed by all network members, exposing the view described in this text. The Manifesto is available at http://www.flosscc.org.

Michael Tiemann, President of the Open Source Initiative and Vice President of Open Source Affairs at Red Hat, confirmed that: "This unique initiative will further enhance FLOSS initiatives in a sustainable manner world wide. All together the Competence Centres will form a network which will enable to share experiences about FLOSS acquired from different perspectives, different cultures and different visions. And all final results will be made freely and openly available to the entire FLOSS community by people talking the same language and sharing the same problems. This represents a notable step towards a true global knowledge economy."

\section{Conclusion}

By sharing a common ethics and culture of collaboration, Competence Centres promote synergies among educational institutions, industry, government, and communities, helping the dissemination and application of knowledge on open standards and technologies, and promoting the development of Information Technology in a way that benefits the entire human society. Furthermore, Competence Centres aim to stay one step ahead in FLOSS market and trends, providing a point of contact among the industrial, academic, and community parts of the FLOSS movement, encouraging the effective use of FLOSS technologies. In short, Competence Centres have the ambition to be significant players of an Information Society based on knowledge sharing. 